\title{
Vivências e estágios na realidade do Sistema Único de Saúde na formação em Odontologia: relato de experiência
}

\author{
Francenilde Silva de Sousa*; Jessyara Brian dos Santos Rêgo** \\ * Cirurgiã-dentista pela Universidade Federal do Maranhão \\ ** Cirurgiã-dentista pela Universidade Federal do Piauí
}

Recebido: 18/06/2021. Aprovado: 22/09/2021.

\begin{abstract}
RESUMO
O conhecimento de como funciona, na teoria e na prática, o sistema público de saúde brasileiro deve ser disseminado aos estudantes durante a sua graduação. Na Odontologia, o modelo flexneriano influenciou a formação dos graduandos por décadas, contudo, após revisões das Diretrizes Curriculares Nacionais do curso, reiterou-se a necessidade da formação voltada para o Sistema Único de Saúde e do perfil generalista do cirurgião-dentista. Assim, o objetivo deste estudo é relatar experiências das vivências e estágios na realidade do SUS e suas contribuições para formação em Odontologia, em consonância com as Diretrizes Curriculares Nacionais do curso. Com o relato notase um despertar incômodo na formação em Odontologia voltada apenas para atuação em clínica ou consultório, e as vivências interdisciplinares propiciam a adoção de competências que contribuirão para os futuros cirurgiões-dentistas. Assim, existe grande cooperação para a formação de sujeitos estratégicos na assistência e gestão do sistema de saúde público brasileiro, conforme perfil esperado para o egresso do curso de Odontologia.
\end{abstract}

Descritores: Saúde Pública. Educação em Saúde. Educação em Odontologia.

\section{INTRODUÇÃO}

A partir da Constituição Federativa do Brasil de 1988, saúde passou a ser um direito de todos e dever do Estado ${ }^{1}$. Esclareceu-se melhor quanto a esse direito com as leis orgânicas de saúde, as $n^{\circ}$ 8.080 e nº 8.142 de 1990 e então, o Sistema Único de Saúde (SUS) passou a ser regulamentado ${ }^{2,3}$.

O SUS objetiva identificar e divulgar determinantes sociais da saúde, criar políticas de saúde e dar assistência às pessoas por meio de ações de prevenção, promoção e recuperação da saúde; garante acesso universal, com integralidade e equidade, da atenção primária à terciária; e é administrado considerando o envolvimento e retorno de gestores, profissionais e usuários ${ }^{2,3}$.

Assim, é importante que estudantes de saúde tenham, durante sua formação, o conhecimento de como funciona o sistema público de saúde brasileiro, na teoria e na prática ${ }^{4}$. Idealizando que acadêmicos poderiam ter os saberes dos possíveis cenários que constituem o SUS, o Ministério da Saúde, em conjunto com a Rede Unida, a Rede 
Governo Colaborativo em Saúde, a União Nacional dos Estudantes (UNE) e os Conselho Nacional de Secretários de Saúde (CONASS) e Conselho Nacional de Secretariais Municipais de Saúde (CONASEMS), formulou um projeto cujo objetivo era inserir os acadêmicos na realidade do SUS $^{5}$.

O piloto desse projeto foi chamado de Escola de Verão e ofertado pela Escola de Saúde Pública do Rio Grande do Sul. Posteriormente, foi expandido às demais instituições de ensino superior (IES) de todo o Brasil para propiciar o debate nas discussões sobre questões sociais, gestão e assistência à saúde e denominado de vivências e estágios na realidade do SUS, o VERSUS $^{5}$.

A necessidade da implantação do projeto surgiu a partir da análise da excessiva especialização de cursos, deficiência no atendimento humanizado, desconhecimento acerca da gestão e administração em saúde e muitas falhas no exercício das equipes interdisciplinares ${ }^{6-8}$. As revisões nas Diretrizes Curriculares Nacionais (DCN) já foram grande avanço, permitindo a flexibilização na formulação de projetos pedagógicos para ajustes na relação de profissionais especialistas e generalistas, sem redução na qualidade da formação $0^{6,9}$.

No âmbito da Odontologia, o modelo flexneriano influenciou a formação de graduandos por décadas ${ }^{10}$, com o enaltecimento de práticas clínicas $^{11}$, atendimento odontológico individual ${ }^{12}$, assistência especializada e mercado privado ${ }^{13}$. Em 2021, ocorreu uma revisão nas DCN do curso de Odontologia na qual reiterou-se necessidade do perfil generalista do cirurgião-dentista; da formação baseada no contexto social, cultural, econômico e ambiental da comunidade em que a instituição está inserida; e da atenção à saúde, tomada de decisão, comunicação, liderança, gestão e educação permanente serem competências dos futuros cirurgiões-dentistas ${ }^{14,15}$.
Diante desse cenário, o objetivo deste estudo é relatar experiências das vivências e estágios na realidade do SUS e suas contribuições para formação em Odontologia, em consonância com as DCN do curso.

\section{RELATO DE EXPERIÊNCIA}

O relato de experiência é resultado de duas imersões teórico-práticas de vivências realizadas em Bacabal, um município do estado do Maranhão. A seleção foi separada em duas etapas: um questionário acerca de questões sociais, econômicas e demográficas e uma carta-intenção, na qual se deveria expor motivos pelos quais o candidato gostaria de participar do projeto.

$\mathrm{O}$ interessado deveria se inscrever e responder o questionário por meio de um site do VER-SUS (http://www.otics.org/estacoes-deobservacao/versus) e, posteriormente, seguir as instruções do edital também disponível no site. $\mathrm{O}$ único pré-requisito para se submeter à seleção era estar matriculado ao menos no $3^{\circ}$ período do seu curso, comprovado com o histórico escolar. A imersão entre 7 a 15 dias, a depender da escolha de cada comissão organizadora. Hospedagem, alimentação e transporte para idas in loco eram custeados pelo Ministério da Saúde.

A primeira edição do VER-SUS Bacabal ocorreu durante dez dias, de quatro a 13 de janeiro de 2016. Foram selecionados 35 participantes, sendo: 30 viventes, os que estavam conhecendo o projeto pela primeira vez e vivenciavam diretamente todas as atividades; e cinco facilitadores, os que conduziam as atividades. A comissão organizadora era formada por sete discentes e uma docente da instituição pública de ensino superior estadual da cidade (Universidade Estadual do Maranhão - UEMA, campus Bacabal). Enfermagem, Educação Física, Fisioterapia, Nutrição, Psicologia, Serviço Social, Medicina, Saúde Coletiva e Odontologia foram os cursos contemplados e representados ao menos com um 
estudante.

A segunda edição ocorreu durante oito dias, de 16 a 23 de janeiro de 2018. Composta por 40 participantes, sendo: 35 viventes e cinco facilitadores. Além de seis discentes e uma docente da comissão organizadora, todos da UEMA. Também existiam representantes dos mais variados cursos da área da saúde para destacar o caráter interdisciplinar da imersão. Nesta edição, foram três estudantes representando o curso de Odontologia: duas viventes e uma facilitadora.

A proposta do VER-SUS envolve o debate entre acadêmicos sobre novas relações e campos de atuação dentro do SUS, respeitando as particularidades individuais e coletivas de cada curso. Ocorreram em comum nas duas edições e favoreciam tais debates: a recepção no primeiro dia para criação de vínculos e realização de acordos de boa vivência; diversificadas atividades (filmes/documentários, leitura de artigos/relatórios, dinâmicas, visitas in loco) desenvolvidas durante os turnos matutino e vespertino; reuniões, todas as noites, com facilitadores e viventes (plenária); e a devolutiva no último dia para ajustes em futuras edições.

É importante salientar que embora houvesse visitas a estabelecimento similares em edições distintas não se tornavam repetitivas, pois além da composição do grupo ser distinta, vivenciei as experiências ora como vivente, ora como facilitadora. Isso tornou cada edição sui generis.

De início foi realizada uma dinâmica para discussão de temas tais como o que é a Saúde Coletiva e seus núcleos de saberes, a Atenção Primária à Saúde (APS) e suas características, as Redes de Atenção à Saúde (RAS), referência e contrarreferência e interdisciplinaridade. Esse foi o ponto de partida para melhor aproveitamento das vivências e estágios. Segundo Alberti (2014) ${ }^{16}$, dinâmicas em grupos possuem repercussão positiva no desenvolvimento de habilidades e competências socioafetivas e mentais.
Também foram discutidos sistemas de saúde de outros países, tendo como base um documentário que apresentava a experiência de estrangeiros na busca por assistência à saúde. $\mathrm{O}$ debate ocorreu à medida em que foram pontuadas similaridades e distinções dos sistemas entre si e com o SUS. As vivências e estágios foram realizadas nos três níveis de atenção à saúde, para discussão da assistência; no núcleo de planejamento e gestão em saúde, permitindo conhecimentos acerca de liderança, tomada de decisões e afins; e em espaços sociais para melhor compreensão do campo das Ciências Humanas e Sociais e aplicação políticas públicas.

\section{Vivências e estágios na Atenção Primária à Saúde}

Houve visitas a Unidades Básicas de Saúde (UBS) com o acompanhamento de equipes da Saúde da Família (eSF). Por meio de rodas de conversas e diálogos com profissionais e usuários do estabelecimento, notou-se bom relacionamento entre os profissionais das equipes e, também, com profissionais do NASF (Núcleo Ampliado de Saúde da Família), apesar de não estarem fixos nas respectivas UBS; além do tratamento humanizado.

Os profissionais explicaram o funcionamento da unidade, incluindo a organização das famílias adscritas. Houve comentários acerca da falta de materiais de insumo, o que atrapalhava o atendimento e afetava a qualidade do cuidado ofertado; e do medo da realização de certas visitas a domicílios, devido localizações julgadas por eles como perigosas.

Foi discutido ainda o quanto a aprendizagem por meio de vivências, estágios e o trabalho em si na unidade (o real) serviu de aperfeiçoamento da atuação e estava pouco distante do ensino somente teórico em sala de aula (o ideal). Nota-se que, assim como foi discutido por Domingos e colaboradores $(2019)^{17}$, os estágios contribuem de forma importante na formação, em especial em 
Odontologia, devido a visualização da prática interdisciplinar da qual possibilita a criação de conceitos de atenção à saúde, humanização, autonomia e aprendizagem do futuro profissional.

Dentre as informações contrastantes a um bom funcionamento da unidade, notou-se que são comuns ao que já foi apresentado em outros estudos $^{18,19}$. Quanto às outras informações, os saberes dos profissionais que atuam na $\operatorname{APS}^{20-22}$ foram notados por meio da percepção do atendimento humanizado, sob territorialização, ciência da ação de determinantes sociais em saúde e de ações preventivas e longitudinais.

\section{Vivências e estágios na Atenção Secundária à Saúde}

Foram realizadas visitas a vários centros com atendimentos especializados, eu fui encaminhada para o Centro de Especialidades Odontológicas (CEO). As queixas foram similares ao já discutido na literatura ${ }^{23,24}$. Criticou-se a falta de instrumentais e materiais, de manutenção e a falha na contrarreferência para a APS possuir devolutiva. Isso afeta o tratamento do usuário, resultando em déficits no acompanhamento terapêutico.

Como atual modelo de referência para tratamento de transtornos mentais ${ }^{25}$, alguns Centros de Atenção Psicossocial (CAPS) também foram também visitados. No CAPS AD (Centro de Atenção Psicossocial Álcool e outras Drogas), centro proposto a pessoas em uso abusivo/dependência de álcool e outras drogas, os profissionais apresentaram o cotidiano nas suas respectivas ocupações e o dos usuários, com desenvolvimentos de atividades domésticas, combinação de regras para bom convívio, momentos de lazer, exercícios físicos, lanches e dinâmicas que estimulam mente e corpo.

Percebeu-se, a partir das histórias dos usuários, que o acompanhamento, para além de medicamentoso, estende-se a seus familiares. Isso faz com que se sintam satisfeitos com a qualidade dos serviços ofertados. De acordo com Lacerda e Fuentes-Rojas $(2017)^{26}$, o envolvimento familiar e desenvolvimento de atividades coopera com a preservação de laços sociais e reinserção social.

Outro local no qual houve notório contentamento pelo serviço prestado de maneira integrada e eficiência foi o Centro de Referência Especializado para População em Situação de Rua (centro POP). Os usuários desse serviço do município de Bacabal/MA demonstraram satisfação pela boa qualidade dos serviços dos profissionais, assim como os do centro POP de Fortaleza, outro município da região Nordeste ${ }^{27}$.

Foram visitados ainda Centro de Referência de Assistência Social e Centro de Referência Especializada de Assistência Social que, assim como o CAPS e o centro POP, não são comuns durante formação no curso de Odontologia. Contudo são ambientes compostos por usuários, profissionais e gestores dos quais devem ter ciência da necessidade da atenção à saúde bucal e da atuação nestes estabelecimentos para atividades preventivas, por meio de palestras, aplicação de flúor ou afins; ou intervenções, seja por atendimentos realizados em IES, por meio de parcerias em projetos de pesquisa e/ou extensão, ou pelo encaminhamento dos usuários a equipes de saúde bucal na APS ou ao CEO.

\section{Vivências e estágios na Atenção Terciária à Saúde}

Representada pelo único hospital local, as vivências e estágios realizados na atenção terciária à saúde foram prejudicas devido curto período de tempo para desenvolvimento das atividades. Esse ocorrido foi resultado de uma falha de comunicação interna para identificação de quem havia recebido os documentos e estava ciente da realização da visita ao hospital. Embora o espaço físico seja maior do que outras unidades de saúde, essa falha pode ser reflexo de má organização.

Foram realizadas conversas com 
profissionais, gestores e usuários de todos os setores, exceto da Unidade de Terapia Intensiva. Conforme Daneliu (2019) ${ }^{28}$, o processo de trabalho em saúde é pautado na inter-relação, e segundo discussões das percepções de viventes e facilitadores, durante plenárias, estava em carência no naquele hospital.

\section{Vivências e estágios na Gestão em Saúde}

Houve visita à Secretaria Municipal de Saúde na qual foram apresentados todos os profissionais dos setores, bem como funcionamento e demonstração de como eram realizadas ações de planejamento e gestão em saúde do município. Ainda ocorreu uma palestra com um integrante da vigilância sanitária com elucidações, tais como a aplicação de dados epidemiológicos na seleção do uso de recursos financeiros e a possibilidade da atuação de um cirurgião-dentista como gestor.

Outro destaque foi dado para a necessidade do controle social, algo historicamente pouco disseminado na população ${ }^{29}$. Nota-se que, assim como foi vivenciado, a literatura aponta recorrentes falhas quanto ao ensino de gestão em saúde durante a graduação, e para além do curso de Odontologia $^{30,31}$.

\section{Vivências e estágios em Espaços Sociais}

Foram realizadas visitas a lixão, centro espírita, terreiro de umbanda e áreas quilombolas. Foi discutido o quanto espaços sociais devem ser respeitados e podem ser aliados na execução da assistência à saúde e na compreensão de recusas.

$\mathrm{Na}$ conversa com catadores de lixo que moravam no lixão, houve comentários tristes referentes à falta de perspectiva para melhorias da saúde. Não eram vacinados e nem sabiam onde poderiam ir para se imunizar. Quando se machucavam, "se viravam" com curativos improvisados feitos de restos de panos encontrados no lixão.
A ida a um quilombo foi caracterizada por pessoas resistentes que possuíam atenção à saúde, mas por vezes suas tradições eram desrespeitadas por ignorância causada pelo desconhecimento de tais tradições. No centro espírita e terreiro de umbanda notou-se que a espiritualidade é uma aliada que deve ser considerada no processo saúdedoença de um religioso.

Os espaços sociais foram responsáveis por explicitar o núcleo das Ciências Humanas e Sociais e a influência de determinantes sociais na saúde das pessoas. A abordagem socioantropológica, na qual busca a subjetividade e considera o todo sem exclusões ou generalizações, reflete em um olhar humanizado do profissional (no caso, dos futuros

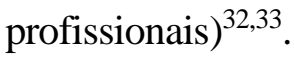

\section{Contribuições na formação em Odontologia}

O projeto contribui para que graduandos se tornem comprometidos com a qualidade $\mathrm{e}$ efetivação do SUS. Experiências em vivências e estágios como esses tendem a influenciar de maneira positiva a formação de futuros cirurgiõesdentistas, como já descrito em outros estudos ${ }^{17,34}$ 36 .

Assim, não seria errôneo instigar um novo modelo de atenção à saúde voltado para a formação, com estágios e vivências que possibilitem aos graduandos a identificação de determinantes sociais; visualização de possibilidades de resolução de problemas; atuação no campo da saúde coletiva; e melhor integração de uma equipe interdisciplinar. O VER-SUS pode ser considerado ponto de partida para elucidação de paradigmas na formação em Odontologia e na saúde como um todo ${ }^{37}$.

Os variados espaços nos quais ocorreram as vivências e estágios interdisciplinares possibilitaram contato com habilidades e competências de um cirurgião-dentista, descritas nas $\mathrm{DCN}^{14}$. Nota-se que experiências similares a esta refletem uma formação voltada para o SUS, 
com grande integração ensino-serviçocomunidade ${ }^{38-40}$.

\section{Limitações e Potencialidades}

Nas plenárias, ao fim do dia de cada vivência, foram discutidas todas as questões relevantes, pontos positivos e negativos e era feita reflexão do quanto aquela experiência poderia contribuir para o graduando. Pelas falas, notou-se a carência dessas experiências na formação em saúde.

Infelizmente, isso foi encontrado em discursos de estudantes de outra região brasileira $^{41}$. Abaixo segue fala de uma vivente de São Paulo, explicitando a falta de conhecimento de todos os núcleos do campo de saber e prática que a saúde coletiva atua e, consequentemente, o profissional pode atuar também.

Conhecia a parte assistencial do SUS. Via inúmeros problemas, mas por desconhecer o sistema em todos os seus segmentos, não encontrava, com clareza, as causas. Não conhecia os eixos de gestão e controle social. ( $F, 23$, enfermagem).

Ademais, ainda suscitaram propostas para aprimoramento da formação em saúde.

Mas isso não é o suficiente, porque sempre seria algo pontual que poucos estudantes teriam acesso (...). Para haver uma mudança efetiva na graduação, os próprios professores precisariam passar por experiências parecidas e terem vontade de realmente fazer diferente. $(M, 20$, fisioterapia).

Um ponto forte, além das discussões que possibilitavam falas de todos, a heterogeneidade das tarefas demonstra o quanto devem ser praticadas vivências e estágios na realidade local em que os estudantes estão inseridos. Isso vai refletir na compreensão do usuário dos serviços de saúde como um indivíduo completo, biopsicossocial e espiritualmente. Ademais, ainda agrega conteúdo ao que cada um conceitua saúde ${ }^{42}$.

\section{CONSIDERAÇÕES FINAIS}

O VER-SUS nasce como estimulante no que tange uma formação dinâmica e interativa com a saúde coletiva em contato direto com a realidade. Os estágios e vivências na realidade do SUS são responsáveis por despertarem um efeito de incômodo da formação em Odontologia voltada para assistência em consultórios do setor privado.

É preciso abranger todos os núcleos da saúde coletiva, em especial por meio de vivências e estágios que possibilitam experiências que desconstruam viseiras, contribuindo para uma formação de sujeitos que podem ser estratégicos para potencializar a organização e gestão do SUS, além de aliados na militância pela sua defesa.

\section{AGRADECIMENTOS}

Ao Ministério da Saúde e suas parcerias para desenvolvimento do projeto VER-SUS e às comissões organizadoras das duas edições do VER-SUS Bacabal/MA, agradecemos imensamente pelas experiências e contribuições para a formação em Odontologia.

\section{ABSTRACT \\ Experiences and internships in the reality of the Unified Health System in Dentistry education: an experience report}

Knowledge of how the Brazilian public health system works in theory and practice should be disseminated to students during their graduation. In Dentistry, the Flexnerian model influenced the training of undergraduates for decades. However, the need for training focused on the Unified Health System and the dentist's generalist profile was reiterated after revisions to the National Curriculum Guidelines for the course. Thus, this study aims to report experiences and internships in the reality of the Unified Health System and their contributions to Dentistry education, aligned with the National Curriculum Guidelines of the 
course. The report reveals the uncomfortable awakening in Dentistry education focused only on clinic or office work, and the interdisciplinary experiences provide the adoption of skills that will contribute to future dentists. Thus, there is significant cooperation for forming strategic subjects in the care and management of the Brazilian public health system, as per the expected profile for Dentistry graduates.

Descriptors: Health Education. Education, Dental. Public Health

\section{REFERÊNCIAS}

1. Brasil. Constituição da República Federativa do Brasil. Brasília: Senado Federal, Coordenação de Edições Técnicas; 1988 p. 498.

2. Brasil. Lei $\mathrm{n}^{\circ} 8.142$, de 28 de dezembro de 1990. Dispõe sobre a participação da comunidade na gestão do Sistema Único de Saúde e sobre as transferências intergovernamentais de recursos financeiros na área da saúde e dá outras providências. Brasília; 1990. 2 p.

3. Brasil. Lei $\mathrm{n}^{\circ}$ 8.080, de 19 de setembro de 1990. Dispõe sobre as condições para a promoção, proteção e recuperação da saúde, a organização e o funcionamento dos serviços correspondentes e dá outras providências. Brasília; 1990. 13 p.

4. Dias GL, Camponogara S, Da Costa VZ, Cezar-Vaz MR, Weiller TH, Cardoso LS. Social representations on health and environment for the family health strategy teams. Saude Soc. 2018;27(1):163-74.

5. Brasil. Ministério da Saúde. Secretaria de Gestão do Trabalho e da Educação na Saúde. Departamento de Gestão da Educação. Ver-SUS/Brasil: cadernos de textos. Brasília: Ministério da Saúde; 2004. $304 \mathrm{p}$.

6. Batista CB. Movimentos de reorientação da formação em saúde e as iniciativas ministeriais para as universidades. Barbarói. 2013;(38):97-125.

7. Brasil. Ministério da Saúde. Ministério da Educação. PRÓ-SAÚDE: Programa Nacional de Reorientação da Formação Profissional em Saúde. Editora do. Brasília; 2007. 78 p.

8. Batista MJ, Gibilini C, Kobayashi HM, Ferreira LL, Silva C, Rosário L. Relato de experiência da interação entre universidade, comunidade e Unidade de Saúde da Família em Piracicaba, SP, Brasil. Arq Odontol. 2010;46(3):144-51.

9. Costa DAS, da Silva RF, Lima VV, Ribeiro ECO. Diretrizes curriculares nacionais das profissões da Saúde 2001-2004: análise à luz das teorias de desenvolvimento curricular. Interface Comun Saúde Educ. 2018;22(67):1183-95.

10. Brasil. A aderência dos cursos de graduação em Enfermagem, Medicina e Odontologia às Diretrizes Curriculares Nacionais [Internet]. Ministério da Saúde, editor. Brasília; 2006. 162 p. [Acesso em 22 set. 2021]. Disponível em: http://abeno.org.br/ arquivos/downloads/download_201112151 03241.pdf.

11. Toassi RFC, Ely LI. Integração entre currículos na educação de profissionais da saúde: a potência para educação interprofissional na graduação. Interface Comun Saúde Educ. 2018;22(supl. 2):156375 .

12. Toassi RFC, Stobäus CD, Mosquera JJM, Moysés SJ. Curriculo integrado no Ensino de Odontologia: novos sentidos para a formação na área da saúde. Interface Comun Saúde Educ. 2012;16(41):529-42.

13. Silveira JLGC, Garcia VL. Mudança curricular em odontologia: significados a partir dos sujeitos da aprendizagem. Interface Comun Saúde Educ. 
2015;19(52):145-58.

14. Brasil. Ministério da Educação. Conselho Nacional de Educação. Câmara de Educação Superior. Resolução $n^{\circ} 03$ de junho de 2021. Institui as Diretrizes Curriculares Nacionais do curso de graduação em Odontologia e dá outras providências. 2021. [Acesso em 22 set. 2021]. Disponível em: http://portal.mec .gov.br/index.php?option=com_docman\&v iew=download\&alias=191741-rces003-21

\&category_slug=junho-2021-pdf\&Itemid= 30192.

15. Fonsêca GS, Junqueira SR, Araújo ME De. Modelo lógico-ideal para o estágio curricular supervisionado: a educação pelo trabalho na formação odontológica. Rev ABENO. 2015;15(2):2-11.

16. Alberti TF, Abegg I, Costa MRJ, Titton M. Dinâmicas de grupo orientadas pelas atividades de estudo: desenvolvimento de habilidades e competências na educação profissional. Rev Bras Estud Pedag. 2014;95(240):346-2.

17. Domingos PAS, Nonato CN, Felício CM. Estágio supervisionado em Odontologia: relato de experiência. J Res Dent. 2019;7(2):18-23.

18. Pereira Limão N, Cesar Campos Ferreira Filho J, Polline Lacerda Protásio A, Marques Santiago B, Bezerra Gomes L, dos Santos Machado L, et al. Dental equipments and supplies and their association with primary health care units. Rev Bras Promoç Saúde. 2016;29(1):84-92.

19. Cunha MS, Sá MC. A visita domiciliar na estratégia de saúde da família: Os desafios de se mover no território. Interface Comun Saúde Educ. 2013;17(44):61-73.

20. Oliveira MPR, Menezes IHCF, Souza LM, Peixoto MRG. Formação e qualificação de profissionais de saúde: fatores associados à qualidade da atenção primária. Rev Bras Educ Med. 2016;40(4):547-59.

21. Carneiro Neto JN, Cordeiro TMSC, Falcão MML. Humanização em saúde e a odontologia. Brazilian J Heal Res. 2015;16(2):130-8.

22. Araújo GB, Filho FWPA, Santos RS, Lira RCM. Territorialização em saúde como instrumento de formação para estudantes de Medicina: relato de experiência. SANARE Rev Políticas Públicas. 2017;16(1):124-9.

23. Rios LRF, Colussi CF. Avaliação normativa dos Centros de Especialidades Odontológicas, Brasil, 2014. Saúde Debate. 2019;43(120):122-36.

24. Goes PSA, Figueiredo N, Neves JC, Silveira FMM, Costa JFR, Pucca GA, et al. Avaliação da atenção secundária em saúde bucal: Uma investigação nos centros deespecialidades do Brasil. Cad Saude Publica. 2012;28(supl):81-9.

25. Brasil. Ministério da Saúde. Secretaria de Atenção à Saúde. Departamento de Atenção Especiailzada e Temática. Centros de Atenção Psicossocial e Unidades de Acolhimento como lugares da atenção psicossocial nos territórios: orientações para elaboração de projetos de construção, reforma e ampliação de CAPS e de UA [Internet]. Brasília: Ministério da Saúde; 2015. 44 p. [Acesso em 22 set. 2021]. Disponível em: www.saude.gov.br/bvs.

26. de Barros Lacerda C, Fuentes-Rojas M. Meanings attributed to Psychosocial Care Center - Alcohol and Other Drugs (CAPS AD) by its users: a case study. Interface Commun Heal Educ. 2017;21(61):363-72.

27. Sousa LLN, Marques MEPS, Bandeira VC, Nogueira VS. Nem mendigo, nem pedinte, cidadãos de direito: um estudo sobre os impactos do centro POP na realidade das pessoas em situação de rua. Rev Gestão 
Desenvolv. 2017;2(1):1-3.

28. Daneliu JL, Santos DVD, Stefanello S, Oliveira VG, Albuquerque GSC. The perception of workers about the work process in a Federal University Hospital under the management of a state-owned private law firm. Saúde Debate. 2019;43(121):378-89.

29. Rolim LB, Cruz RSBLC, Sampaio KJAJ. Social control and popular participation as a guideline of SUS: a narrative review. Saúde Debate. 2013;37(96):139-47.

30. Leonel MS, Cruz MS, Lopes JS, Rosa WVS. Management in Public Health and Nursing graduations: an analysis of the political-pedagogical projects. Gestão Saúde. 2020;11(2):161-77.

31. Cunha MLS, Hortale VA. Characteristics of health management courses in Brazil. Saúde Debate. 2017;41(113):425-40.

32. Minayo MC de S. O desafio do conhecimento. In: Pesquisa qualitativa em saúde. 1 ed. São Paulo: Hucitec - Abrasco; 1992. p. 269.

33. Carrapato P, Correia P, Garcia B. Determinante da saúde no Brasil: a procura da equidade na saúde. Saúde Soc. 2017;26(3):676-89.

34. Kammer PV, Hofstaetter V, Sorgato EDFF, Steglich M, Almeida BS de, Luz MFS da, et al. Inserção de estudantes de Odontologia no SUS: relato de experiência. Extensio. 2019;16(34):103-13.

35. Viudes Bruder M, Fernando Lolli L, Rosa Palácios A, Barbosa da Rocha N, Cristina Veltrini V, Gasparetto A, et al. Estágio supervisionado na odontologia: vivência da promoção da saúde e integração multiprofissional. Rev Bras Promoç Saúde. 2017;30(2):294-300.

36. Mafi A, Moretto C, Teixeira MFN, Saldanha OMFL, Rados ARV. A interdisciplinaridade e seus reflexos na formação do cirurgião-dentista. Rev ABENO. 2017;17(1):62-73.

37. Maranhão T, Matos IB. Vivências no sistema único de saúde (SUS) como marcadoras de acontecimento no campo da Saúde Coletiva. Interface Comun Saúde Educ. 2018;22(64):55-66.

38. Silveira JLGC, Kremer MM, Silveira MEUC, Schneider ACTC. Perceptions of teaching-service-community integration: contributions to health education and to comprehensive healthcare. Interface Comun Saúde Educ. 2020;24:e190499.

39. Mendes FMS, Fonseca KA, Brasil JA, Dalbello-Araújo M. Ver-SUS: relato de vivências na formação de Psicologia. Psicol Ciência e Profiss. 2012;32(1):174-87.

40. Ferla AA, Maranhão T, Rocha CMF, Vera R, Peixoto GP, Silva IF, et al. As histórias das vivências e estágios no Sistema Único de Saúde em seus múltiplos cenários. In: Múltiplos cenários do VER-SUS: vivências e estágios de Norte a Sul do Brasil. Rede Unida. Porto Alegre; 2016. p. 8-10.

41. Araújo D, Miranda MCG de, Brasil SL. Formação de profissionais de saúde na perspectiva da integralidade. Rev Baiana Saúde Públ. 2007;31(supl. 1):20-31.

42. Stronks K, Hoeymans N, Haverkamp B, Den Hertog FRJ, Van Bon-Martens MJH, Galenkamp H, et al. Do conceptualisations of health differ across social strata? A concept mapping study among lay people. BMJ Open. 2018;8:e020210.

\section{Correspondência para:}

Francenilde Silva de Sousa e-mail: fraansoousa@gmail.com Coordenação de Odontologia Avenida dos Portugueses, 1966 Vila Bacanga 65080-805 São Luís/MA 